\title{
From Composites to Service Systems: The Role of Emergence in Service Design
}

\author{
Anshuman Saxena ${ }^{1,2}$ \\ ${ }^{1}$ Systemic Modeling Laboratory, I\&C, EPFL Switzerland \\ ${ }^{2}$ TCS Innovation Labs, Bangalore India \\ anshuman.saxena@\{epfl.ch,tcs.com
}

\author{
Alain Wegmann \\ Systemic Modeling Laboratory (LAMS) \\ I\&C, EPF-Lausanne, CH 1015 Switzerland \\ alain.wegmann@epfl.ch
}

\begin{abstract}
Service Design is an engineering endeavor to enrich some aspect of the real world through a man-made artifact. Systems approach to modeling reality is particularly relevant to Service Design as it seeks to explain reality as a composition of functional observations. Nevertheless, its adoption varies from casual interpretations of interconnectedness to citations of nondeducible causality. To establish Systems approach firmly within the domain of Service Design, it is important to provide an unambiguous characterization of the nature of composition that a Systemic view entails. Further, such characterization should be amenable to the development of a formal framework for specifying Services. In this paper, we take a cognitive approach to composition and highlight the difference between composites as structure-unifying integrated-wholes and composites as emergence-revealing systems. We then translate this characterization into a set of visual semantics for expressing a service-oriented view of observed reality.
\end{abstract}

Keywords-compositional hierarchy; behavioral discontinuity; design patterns

\section{INTRODUCTION}

Unlike the goods-centered model of economic exchange, where goods are seen as "physical embodiments of competence" [1] and are marketed as potential source for benefits [2], services focus on the actual benefits experienced how the adoption of some man-made artifact enriches the real world [3]. Uncertainties of the adoption process and the tacit nature of consumption context make the information relevant for service design hard to model and, most often, sticky to quantify [4]. Further, as formalized in the Law of Requisite Variety [5], for a design to deliver desired results in a given situation, the design must possess an amount of variety that is at least equal to the variety that the situation can present. As a result, a service designer is required to cast her net wide and try to understand the different facets of the interactions that the phenomenon of interest exhibits with its environment.

A Systems view of observed reality corresponds to levels of abstraction, which best reflect the modeler's view of the interactions between the phenomenon being modeled and its environment. Nevertheless, these levels of abstraction may not always be primary in nature in the sense that they may not correspond to the modeler's first/immediate view of the reality. The modeler explores reality at multiple levels of abstraction the additional levels being the result of an explicit composition from primary observations. The basis for such composition can vary from casual interpretations of interconnectedness to citations of non-deducible causality. From a combinatorial perspective, an unqualified compositional approach would yield an exponentially large set of composites, not all of which may be relevant to explaining the causal organization of the observed reality. The relevance of an abstraction level in explaining the causal organization of the observed reality can be formalized in terms of the behavioral-novelty that it reveals. An explicit admission of such novel properties as part of the service specification increases the amount of variety embedded in the service thereby increasing the likelihood that the service yields desired benefits.

In this paper, we take a cognitive approach to composition and provide an unambiguous characterization of the nature of composition that a Systemic view entails. The focus is on clarifying the distinction between the two types of composites that are most usually confused and, hence, used interchangeably - structure-unifying composites and emergence-revealing composites. Further, to make this conceptualization of Systems useful for service designers, we propose a set of visual semantics that can help them in expressing a service oriented view of the phenomenon of interest.

The remaining part of this paper is structured as follows: Section 2 presents a survey of the related work - describing, in particular, the cognitive groundings that underlie Systems conceptualization; Section 3 focuses on the organization of observations as compositional hierarchies, providing a characterization of composition that is aimed at identifying behavioral-novelty revealing levels in the hierarchy; Section 4 translates this characterization into a set of visual semantics useful for the early phase of Requirements Engineering. The concepts introduced in this paper are illustrated through an inquiry aimed at enriching the phenomenon of "Bike as a means of transport" through the creation of an IT enabled mobility management service. The example, though trivial at first sight, provides enough richness to illustrate both the challenges that a Systems conceptualization faces and the merits of the approach proposed in this paper. The paper ends with some concluding remarks in Section 5.

\section{FUNDAMENTALS OF SySTEMIC CONCEPTUALIZATION}

Systems are an important conceptualization in an inquiry. They correspond to levels of abstraction, which best reflect the modeler's view of the interactions between the phenomenon being modeled and its environment. Nevertheless, the 
modeler's viewpoint is not formed in isolation. The prior experience of the modeler influences the inquiry through the admission of various heuristics and biases [6]. From a cognitive perspective, the background knowledge of the modeler is organized in her mental space around different cognitive themes, referred to as idealized-cognitive models (ICMs), such as the ones grounded in visual perception, inter-domain communication, knowledge organization, and functional behavior [7]. These models are not mutually exclusive. A first conceptualization benefits from an automatic, unconscious and simultaneous activation of several such models yielding basiclevel categories [8]. These categories are basic in that they represent an ontological reification of the innate functional and epistemological view of the modeler.

The basic-level conceptualization, though primary, may not suffice for the purpose of an inquiry. For example, a serviceoriented inquiry, which seeks to enrich a real world phenomenon through the creation of some man-made artifact, needs to understand how the observed reality is causally produced. This includes gathering information about both what constitutes reality - the ontological categories observed by the modeler [9], and how these categories interact to functionally produce the observed reality. Basic level categories represent a level of abstraction at which the modeler's observation exhibits maximum correlation with her ICMs. ICMs bring to bear a combination of several perspectives - functional behavior being only one of them. As a result, basic level categories by themselves may not always be adequate to explain the causal production of observed reality. Nevertheless, they present an ideal starting point for the modeler to refine her view by exploring reality at additional levels of abstraction.

Refinement is the process of identifying mappings between different levels of abstraction [10]. Ontological refinement seeks to refine an ontological category, [11], into a set of lower level categories such that the resulting categories contribute to the semantics of the higher-level category. In the context of modeling reality, any ontological refinement is subject to the modeler's assignment of semantics to the mapping between the different levels of abstraction, which, in turn, is an outcome of the invocation of his ICMs and, hence, not exclusively functional in nature.

For example, consider an inquiry aimed at enriching the phenomenon of <<Bike_as_a_means_of_transport $>>$ through the creation of an IT enabled mobility management service. The inquiry may start by revealing the following basic level categories: <<Bike>> as a mechanical assembly, $<<$ Road $>>$ as a friction providing surface for traversal, and $<<$ Rider $>>$ as a human capable of balancing and propelling the bike. In an effort to understand the causal organization of these categories, the modeler refines her basic level observations to lower levels of abstraction. Cognitively, the process of refining categories calls for repeated invocation of ICMs, each iteration generating categories with finer details. Given our general familiarity with bikes, one might be inclined to suggest an ontological refinement of $<<B i k e>>$ that not only identifies its constituent parts but also reveals how these parts can be composed to mechanically assemble the bike. Nevertheless, functional behavior is only one of the several cognitive groundings that motivate ICMs. To ensure the

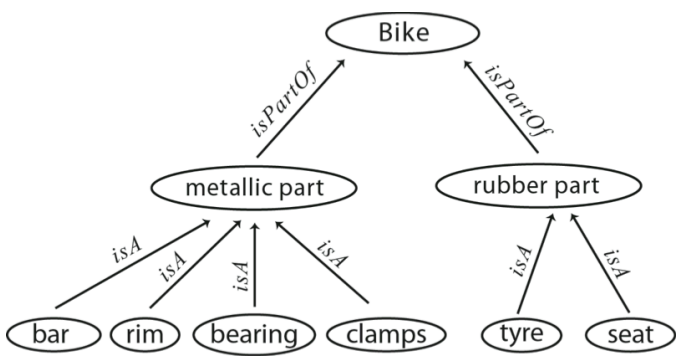

Figure 1. A representative refinement of Bike as a mechanical assembly

generality of our argument beyond the current exposition, let us assume that the modeler's view is shaped by a combination of ICMs. As a manifestation of this influence, the modeler might see bike as composed of metallic and rubber parts, which in turn are specialized into - bar, clamps, bearings and tyre. Fig. 1 presents a hierarchical depiction of the refinement of the category $<<$ Bike $>>$. Edges tagged isPartOf signify decomposition and the ones tagged is $A$ signify specialization. The mixed semantics of refinement in this example suggests that not all ontological refinements may lead to purely compositional hierarchies. For instance it is not clear how $<<$ rim $>>$, <<bearing $>>$ and <<tyre > come together to form a functional sub-assembly, say wheel, or if there exist any functional sub-assemblies at all.

Ontological refinement enriches the modeler's view of reality by admitting observations at different levels of abstraction. The expanded set of categories can be reorganized in a way that the composites, obtained by combining one or several of these categories, contribute to some functional aspect of observed reality. The principle of nearly complete decomposition [12], which states that for a given phenomenon of interest, the interactions between the constituent categories are not all of equal strength - some interactions are stronger than the other, suggests that the resulting composites can be structured as a hierarchy. From a combinatorial perspective, an unqualified compositional approach would yield an exponentially large set of composites, not all of which may be conceptually relevant to the modeler, let alone contribute to her understanding of the causal organization of the observed reality. It is, therefore, important that the modeler can distinguish between different kinds of composition and choose the one that is most relevant to explaining the causal organization of the observed reality. Fig. 2 shows a representative compositional hierarchy for $<<\mathrm{Bike}>>$ with $<<$ wheel $>>$ and $<<$ frame $>>$ as functional sub-assemblies. It also shows some additional composites, which are either conceptually irrelevant to the modeler or simply beyond the scope of the current phenomenon of interest.

Even in compositional hierarchies where each composite has some functional relevance to the causal organization of the observed reality, there can be discontinuities between the behaviors revealed at different levels of the hierarchy. A behavioral discontinuity refers to the non-trivial deducibility of higher-level behavior from behaviors exhibited at the lowerlevels. The occurrence of behavioral discontinuity in a compositional hierarchy is an indication of some qualitatively 


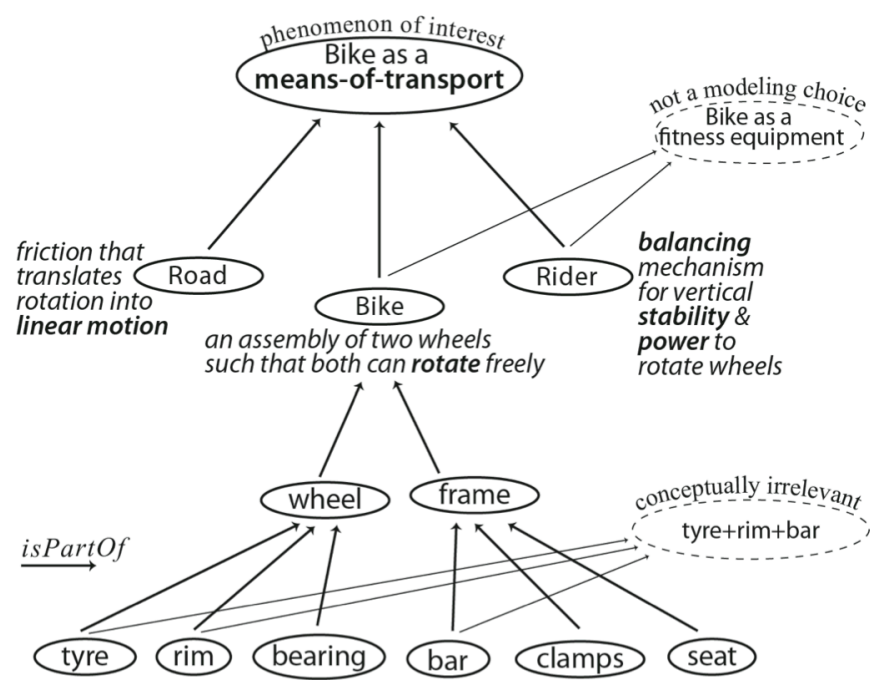

Figure 2. A compositional hierarchy of Bike as a means of transport

novel property of the observed reality - one that cannot be established at any of the lower levels of the hierarchy. The field of Complexity Science refers to such properties as emergent properties and the occurrence of associated behavioral discontinuity as the phenomenon of emergence.

From an informational perspective [13], levels revealing functionally novel aspects of the observed reality are critical to the modeler's understanding of how the phenomenon of interest interacts with its environment. A Systems approach to inquiry emphasizes on identifying levels of abstraction where such novelty occurs. Fig. 2 shows the emergence of means-oftransport as a functionally novel property. Here the evidence for the occurrence of behavioral discontinuity is linguistically inspired and rests in the sudden change of vocabulary between the different levels of the hierarchy: rotation, linear motion, power, stability and balance at the lower level and means-oftransport at the higher level. Fig. 3 summarizes the different stages in the deveopment of a Systemic conceptualization of observed reality.

\section{THE NATURE OF SySTEMIC COMPOSITION}

The modeler's view of a given phenomenon of interest is composed of her observations about reality at multiple levels of abstraction. The ontological categories representing such observations can be combined to explain the causal organization of the phenomenon of interest. Nevertheless, an unqualified compositional approach would yield an exponentially large set of composites - the power set of the set of categories. Not all of them may be conceptually relevant to the modeler, let alone contributing to her understanding of the causal organization of the observed reality. It is, therefore, important that the modeler can distinguish between different kinds of composition and choose the one that is most relevant to explaining the causal organization of the observed reality. The following three types of compositions are particularly interesting in our context.

\section{A. Mereological Sum}

A mereological sum is a mechanical aggregation of categories, which may or may not have any conceptual relevance to the modeler. Such an aggregation is inspired by an absolute connectionist view of the world where everything is connected to everything with equal strength. In real world some connections are stronger than the others and, hence, any arbitrary combination may not always have noticeable conceptual relevance to the modeler. As noted in [14], an example of a mereological sum can be "Noam Chomsky's left foot, the first act of Puccini's Turandot and the number 3", which from a combinatorial perspective is a perfectly legitimate entity, though it may be extremely hard to find a context to which it can be conceptually relevant. In the context of the bike example, Fig. 2 shows, in addition to conceptually relevant categories like <<wheel>> and <<frame>>, an aggregation of tyre, rim and bar, which does not contribute to the modeler's conceptualization of bike.

It is important to note that not all aggregates can be organized as a compositional hierarchy. Unlike the absolute connectionist view of equally-strong-association-between-all, hierarchy theory promotes a connectionist view based on the comparative-strength-of-associations [15]. It is this ability to compare the relative strength of aggregation that will allow defining a partial order on the aggregates, thereby organizing them in a hierarchy. Comparison is a cognitive exercise and is only feasible if the subjects have some conceptual relevance to the modeler [16]. Thus, only aggregates that have some conceptual relevance to the modeler can be organized as a compositional hierarchy.

\section{B. Integrated Whole}

Integrated-wholes are mereological sums that have some conceptual relevance to the modeler. They represent a composition of categories that is unified under some binding relation [17]. The binding relation is a formal characterization of the conceptual relevance that the integrated-whole holds for the modeler. It is this conceptual relevance that allows the modeler to organize the integrated-wholes as a hierarchy. Hierarchies are defined using a partial order relation based on the relative strength of the part-of relations, aka meronymic relation [18], that produce them and are hence referred to as compositional hierarchies. In addition to the logical properties of transitivity, irreflexivity, and antisymmetry, which define a partial order [19], meronymic relations can have multiple semantic connotations. Three important dimensions along

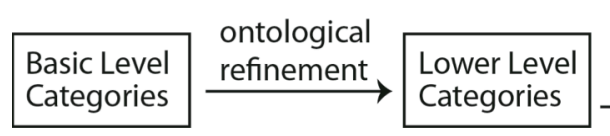

conceptually relevant composition,

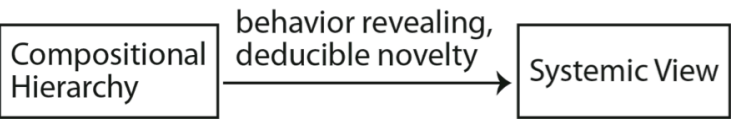

Figure 3. A cognitive perspective of the process for developing a Systemic conceptualization of observed reality 
which a meronymic relation can be characterized are - whether the lower-level categories are functionally related to the higherlevel category, whether the lower-level categories can exist independent of the higher-level categories, and whether the lower and higher-level categories are of the same type [20].

In the context of specifying an engineering design, which is the end objective of the modeler conducting a service-oriented inquiry, the focus is on understanding the functional behavior as perceived in observed reality. Hence, in compositional hierarchies each lower-level category contributes, in some way, to the functionality of the higher-level category.

The independence of categories corresponds to the conceptual uniqueness of the relevance that an integratedwhole has to the modeler. The existence of binding relation as a formalization of the modeler's focus on some specific aspect of the observed phenomenon and the closure of the integratedwhole under this binding relation together, delineate parts of the phenomenon relevant to the aspect under focus from the rest of the environment. Thus, the hierarchical organization of categories is tantamount to decomposing the modeler's view of the observed phenomenon along different aspects of the phenomenon. Each level in the hierarchy presents a level of abstraction, which reflects the modeler's view of the interactions between the phenomenon being modeled and its environment. Since an integrated-whole is modeled as interacting with the environment, it can be interpreted as enjoying an independence of existence. Thus, meronymic relations exhibited by integrated-wholes are the ones where the lower-level categories are functionally related to higher-level categories and can exist independent of the higher-level categories. Such class of meronymic relations is referred to as component-integral object meronymic class [20].

One interpretation of the type of categories is the newness that it brings to the understanding of the causal production of the observed reality. Nevertheless, not all levels of the compositional hierarchy may contribute to any new understanding of the observed behavior, i.e. the interaction of the observed phenomenon and its environment. The notion of newness can be explained both ontologically and epistemologically [21]. From an ontological point of view, newness is the occurrence of qualitative novelty; say, a property that exists at one level of abstraction but not at any of the lower levels. In the context of compositional hierarchies, like the ones detailed above, the adoption of binding relation as a characterization of integrated-whole might seem to suggest the existence of behavior-revealing qualitative novelty. Nevertheless, the conceptual relevance, which defines each binding relation, can be inspired by variety of considerations not all of which may be grounded in behavioral semantics. For example, a large class of binding relations is based on the Gestalt effect [22], which is the form generating capability of the modeler, particularly with respect to the visual recognition of figures and whole forms. The qualitative aspect of the novelty associated with Gestalt effect is structural in nature and may not necessarily correspond to any new understanding of the behavior.

\section{Systems}

Integrated-wholes, which exhibit behavioral-novelty, are referred to as emergent [23] and the behavioral-novelty as an emergent property [24] of the integrated-whole. From an epistemological point of view, newness can also be interpreted from the lack of predictability of the higher level from lower levels. Such a formulation of newness aims to categorize integrated-wholes based on the computational hardship involved in establishing causal relationships between different levels of abstraction.

The ontological and epistemological interpretation of novelty can be combined to provide a general framework for characterizing implementable Systems. To recall, we defined Systems as levels of abstraction, which best reflect the modeler's view of the interactions between the phenomenon being modeled and its environment. The ontological focus on distinguishing behavior-revealing novelty from the structureunifying novelty of integrated-wholes is aimed at identifying precisely those levels of abstraction, which contribute to a better understanding of the behavior of the observed phenomenon. The level exhibiting behavior-novelty and levels below it where this novelty does not exist together constitute a Systemic view of the observed phenomenon [25]. Nevertheless, from a service design point of view, existence of behaviornovelty is not enough. It is important that such novelty can be engineered from the lower level constituents. This calls for an understanding of how the levels below the novelty exhibiting level cause the novelty to emerge. This corresponds to the, above discussed, epistemological view of novelty, which can be framed as the amount of computation required in establishing causality of emergence. Novelty with finite computational demands on establishing its causality from lower-levels is deemed deducible, while open-ended demands on computing causality is considered non-deducible. From a service design perspective, levels of abstraction, which correspond to deducible behavioral-novelty are a more meaningful representation of systems constituting an observed phenomenon as the can be engineered in man-made artifacts. The deducible form of behavior-novelty is referred by many as the weak form of emergence, [26], and the non-deducible form of behavior-novelty as the strong form of emergence, [27]. Table 1 presents four different characterizations of the compositions corresponding to a level of abstraction. Integrated-wholes with only structure-unifying novelty are deducible as they result from the binding relation, which itself is a modeler's construction. As for structure-unifying but nondeducible compositions, they correspond to mereological sums and are, from an engineering point of view, an invalid combination since structure-unifying compositions owe their

TABLE I. COGNITIVE CHARACTERIZATION OF COMPOSITION

\begin{tabular}{|l|l|l|}
\hline \multicolumn{1}{|c|}{ Composites } & \multicolumn{1}{|c|}{$\begin{array}{c}\text { Ontological } \\
\text { (Existence of Novelty) }\end{array}$} & $\begin{array}{c}\text { Epistemological } \\
\text { (Nature of Novelty) }\end{array}$ \\
\hline Abstract System & Behavior-revealing & Non-deducible \\
\hline $\begin{array}{l}\text { System } \\
\text { (implementable) }\end{array}$ & Behavior-revealing & Deducible \\
\hline Integrated Whole & Structure-unifying & Deducible \\
\hline Mereological Sum & Structure-unifying & Non-deducible \\
\hline
\end{tabular}


existence to binding relations, which are essentially the causeand-effect deductions of the modeler from lower-level categories.

\section{VISUAL SEMANTICS FOR SERVICE-ORIENTED VIEW}

Modeling reality is the first step in an inquiry. It constitutes the early requirements engineering (RE) phase of service design where stakeholders develop and communicate their view of the observed reality corresponding to some phenomenon of interest [28]. While plurality of observations is vital to uncover different facets of the phenomenon of interest, and should be encouraged, it is important that different views can be reconciled into one unified view of observed reality. It is only once a shared view of reality is established that the project can progress and deliver desired results. In the context of IT service design, the RE phase usually results in a service specification document following some standardized structure like the one prescribed in IEEE 830 [29]. Nevertheless, before such domain specific deliverables can be specified, the stakeholders need to communicate to reconcile their views of observed reality.

Visual narratives are one of the most effective modes of communication at this early stage of design. Stakeholders come from varied domains of activity and visual exchange is usually the most inclusive way of communication. Nevertheless, the understanding of visual perception should not be limited to the literal interpretation of seeing as a visual apprehension of spatial gestalts but also as one, which invokes appreciative judgments of quality [30]. Thus visual design should not only focus on the assignment of symbolic descriptions to rules so as to visually register information, but also the processing of such information to enable the construction of meaning. Visual semantics is the phenomenon of externalizing this process through an explicit admission of meaningful visual patterns that enable the invocation of pre-assigned meanings [31].

In this section we propose a set of visual semantics for enabling a coherent service-oriented view of observed reality.

\section{A. Graphical Notation}

The concept of integrated-whole is the primary building block in enabling a service-oriented conceptualization. To recall, integrated-wholes are a mapping between reality and the conceptual space of the modeler.

Conceptual spaces, as defined in [32], describe the structure of mental representation at some unique level of abstraction where information from all modalities of experience, such as linguistic, sensory and motor, are compatible [33]. From a cognitive perspective, two other levels of mental representation can be explored to communicate a concept - symbolic level and the connectionist level. Representing a concept at the symbolic level calls for identifying a set of symbols and rules, which govern their manipulation [34]. Understanding a concept is then a combinatorial exercise in symbol manipulation, one that does not lends itself easily to interpersonal communication. Connectionist representations, on the other hand, are high dimensional space of activities where the interpretation of dimensions usually calls for some external measure to reduce them into humanly manageable categories.
Given the overhead involved in understanding a representation modeled at both symbolic and connectionist level, we provide a representation of integrated-whole at the conceptual level. This is an intrinsic approach to representation where the representation exhibits the same relations as what the concepts represent [35], and is hence more intuitive to relate. Integrated-wholes represent a category that is closed under some relation. In a two dimensional space medium like paper, the closure property is represented as a region in conceptual space with its one unique identity. A nonsymmetric graphical notation for depicting an integratedwhole is presented in Fig. 4.

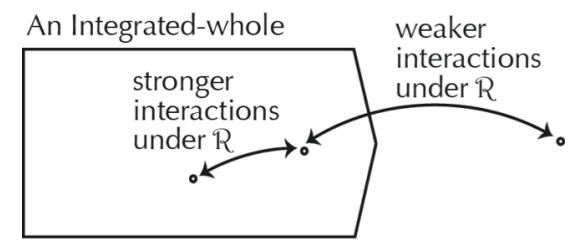

Figure 4. Graphical notation for integrated-whole

\section{B. Design Patterns}

Design patterns are named problem/solution pairs, which codify well-established and proven principles of design in a structured format that facilitates their repeated application to new contexts [36]. We adopt the same format of specifying design patterns as the one used in [37] for describing design patterns for software design.

Pattern Name:
Problem:
Solution:

\section{Whole/Composite}

How to represent a Systemic view of reality? By making explicit the difference in compositional nature of structure-unifying integrated-wholes and emergence-revealing systems.

A systemic view corresponds to behavior-novelty revealing levels of compositional hierarchy. Nevertheless, novelty can only be inferred by contrasting the information available at different levels. It is not possible only by looking at one level to infer if the integrated-whole constitutes a system or not. It is only when levels are seen in pair that one can identify if the higher level exhibits structure-unifying behavior or emergence-revealing behavior. The pattern Whole/Composite is a visual codification of the compositional nature of an integrated-whole. Fig. 5 presents a schema that visually captures the existence of emergent property through an explicit modeling of the process of emergence ( $R$ ') that reveals some behavior-reveling novelty at a higher level. The tag [W] signifies an integrated whole at a level higher in the compositional hierarchy as compared to the one tagged [C], which reveals the composition of the higher level integrated whole from lower level categories. 


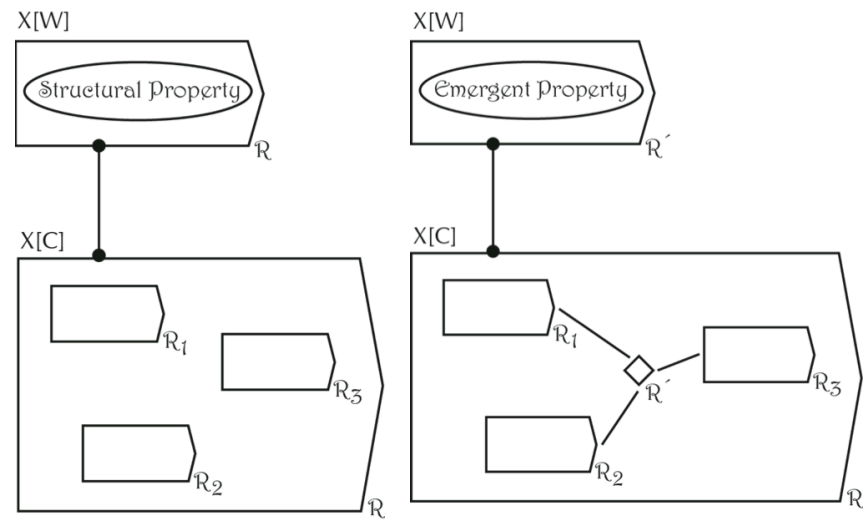

Figure 5. Schema for visual appreciation of Systemic view

Pattern Name: Supplier/Adopter

Problem: How to represent a Service view of reality?

Solution: By making explicit the different roles and responsibilities that the stakeholders undertake to realize benefits for themselves.

Service is the process of realizing benefits to the stakeholders of some phenomenon of interest. Benefit is a subjective notion best explained by the stakeholder herself. As a result, to ensure that a service realizes desired benefits, it is important that the stakeholder is included in the designing of the service. For a service to be sustainable, each stakeholder of the service should be benefited in some desired way. This suggests the duality of roles that each stakeholder undertakes as part of the service design. A stakeholder is both a supplier and an adopter of the service [38].

A service view of observed reality should clearly specify the benefits that the service will bring to each stakeholder of the service, and the roles and responsibilities that need to be undertaken/fulfilled to jointly realize these benefits [39]. These roles and responsibilities can be assigned at different levels of the compositional hierarchy. Taking a systemic view of observed reality helps the service designer to identify levels of activities, which, if ensured, can account for the non-trivial aspects of the observed behavior, thereby increasing the likelihood of realizing desired benefits.

From an engineering point of view, a supplier role signifies some responsibility that a stakeholder undertakes towards the production of some aspect of the service leading to the realization of some benefit to some stakeholder. A grouping of supplier roles, assigned at the systemic level, based on the activities that need to be undertaken to realize a benefit for some stakeholder constitutes a Service System. Since each stakeholder benefits from the service, a service-oriented view should reveal one Service System for each stakeholder in the adopter role. Service System can be seen as work systems [40], striving to realize the benefits for some stakeholder. Fig. 6 presents a schema that visually captures the service-oriented view corresponding to the systemic level R'. A complete

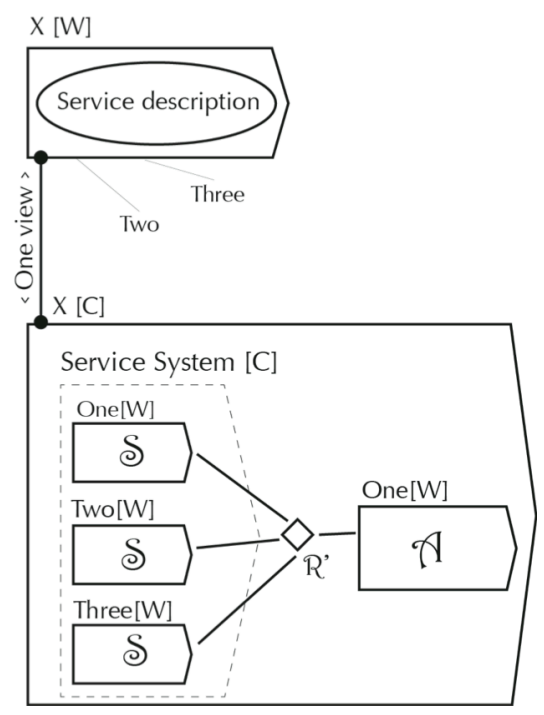

Figure 6. Schema for visual appreciation of Service view Roles : Supplier $₫$ and Adopter $\uparrow$

service description identifies the benefit for each stakeholder of the service and the service systems responsible for realizing those benefits.

\section{Bike example revisited}

In this section we revisit the bike example using the visual semantics presented in earlier sections. Fig. 7 models bike as an integrated-whole composed of all those parts that are permanently connected to each other to give bike its conventional appearance as a mechanical assembly of wheels connected to a frame. The word permanent is to signify extended period of time over which the parts of the bike remain interconnected, as compared to, say, the rider who is connected only while she rides the bike.

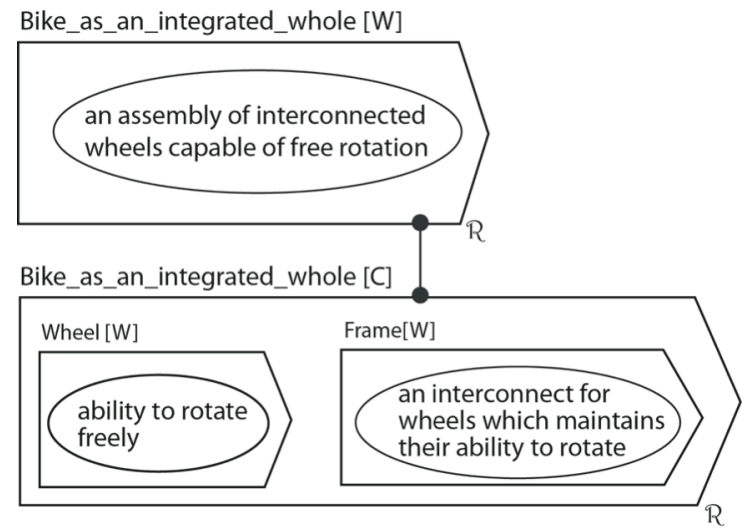

Figure 7. An integrated-whole representation of Bike R: Temporally extended interconnectedness

Fig. 8 models bike as a system, R', exhibiting the emergent property of rideable_means_of_transport. Since every system is also an integrated-whole, in addition to the emergent 
property of the system, we will also see some structure unifying property, like all components in physical contact while the bike is in use, including the rider.

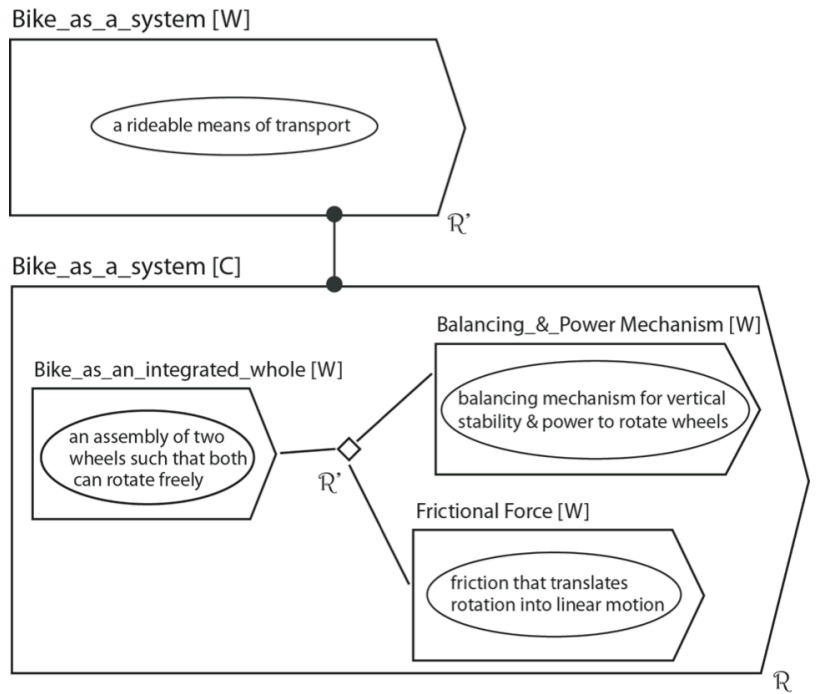

Figure 8. A Systemic representation of Bike $\mathrm{R}$ : Temporally limited interconnectedness; R': Means of transport

Fig. 9 models the service-oriented view of bike. It identifies the stakeholders of bike-as-a-means-of-transport as the Bike Manufacturer, who mechanically assembles the bike; City municipality, which provides ride-able roads; and Ms. She, who rides the bike. All these stakeholders receive some benefit from the Bike-as-a-system. This is captured in service description - Bike manufacturer makes profit by selling bike to Ms. She who uses it as a means of transport by riding the bike on city roads provided by the City municipality for citizen wellness. Each stakeholder then sees the system of Bike-as-a-means-of-transport as providing some service to it. Service systems formalize this view through the grouping of supplier roles for each target benefit. A service-oriented view thus includes multiple views; one for each of the stakeholders - revealing how the benefit they desire is going to be produced.

\section{CONCLUSION}

Systems is a theory of observation. It empowers the modeler to identify aspects of reality that reveal behaviorally novel aspects of the phenomenon of interest. Systems approach to modeling reality is particularly useful in identifying the design requirements for services. Service Design seeks to influence aspects of reality through the creation of man-made artifacts and a systemic conceptualization of reality can help the service designer in identifying non-trivial aspects of reality, which, if preserved, would increase the likelihood that the service delivers desired benefits. This paper is an attempt to make explicit the cognitive process of developing a systemic view of reality. Such an explication can help the modelers to make a conscious effort to look for novelty in the observed reality. We also present a set of visual semantics that can aid the service designers in the development and communication of
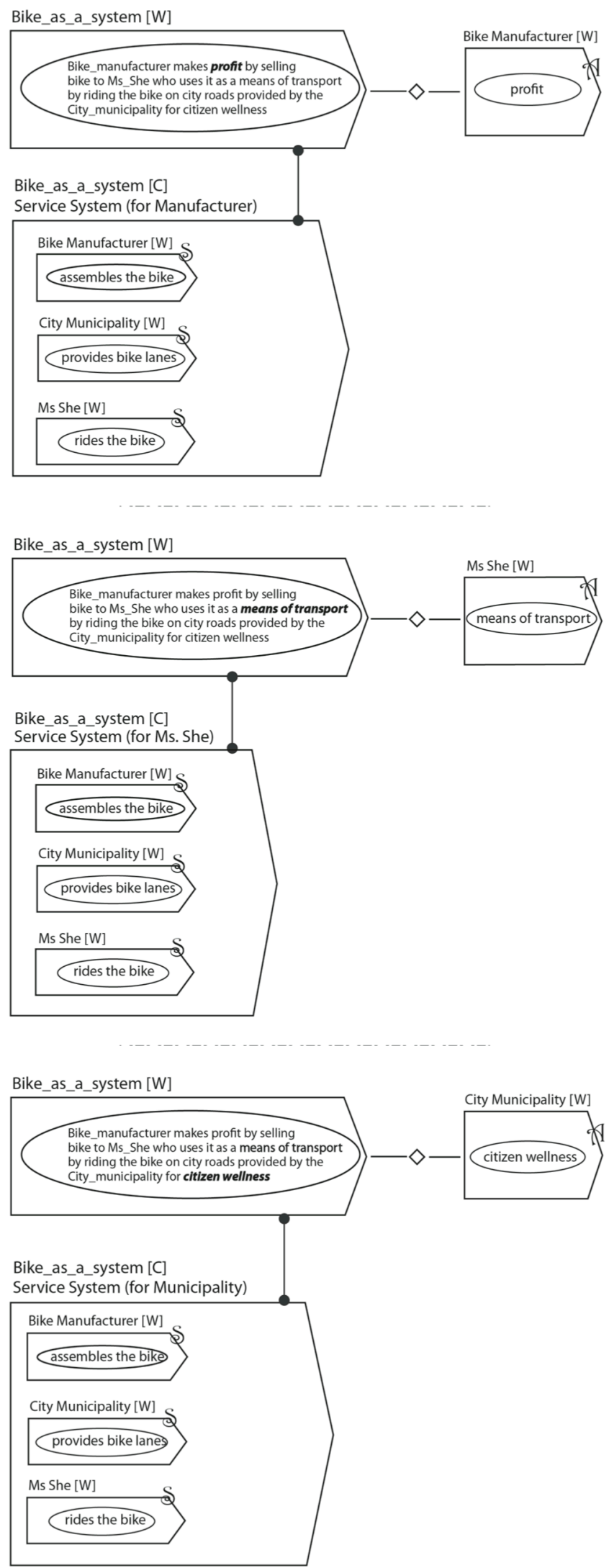

Figure 9. Service-oriented views of the system Bike as a ride-able means of transport 
a systemic view of reality during the early phase of requirements engineering.

\section{REFERENCES}

[1] C. K. Prahalad and G. Hamel, "The Core Competence of the Corporation," vol. 68, A. Read, Ed., ed: Harvard Business School Publication Corp., 1990, p. 79.

[2] S. D. Hunt, Foundations of Marketing Theory: Toward a General Theory of Marketing: M.E. Sharpe, 2002.

[3] S. L. Vargo and R. F. Lusch, "The Four Service Marketing Myths," Journal of Service Research, vol. 6, pp. 324-335, May 1, 20042004.

[4] E. v. Hippel, "Sticky information and the locus of problem solving: implications for innovation," Manage. Sci., vol. 40, pp. 429-439, 1994.

[5] W. R. Ashby, Introduction to Cybernetics: Methuen, 1964.

[6] A. Tversky and D. Kahneman, "Judgment under Uncertainty: Heuristics and Biases," Science, vol. 185, pp. 1124-1131, September 27, 1974 1974.

[7] G. Lakoff, Women, Fire, and Dangerous Things: University Of Chicago Press, 1990.

[8] E. Rosch, C. Mervis, W. Gray, D. Johnson, and P. Boyes-Braem, "Basic objects in natural categories," Cognitive Psychology, vol. 8, pp. 382-439, 1976.

[9] T. R. Gruber, "Toward principles for the design of ontologies used for knowledge sharing," Int. J. Hum.-Comput. Stud., vol. 43, pp. 907-928, 1995.

[10] M. L. Abadi, Leslie, "The existence of refinement mappings," Theor. Comput. Sci., vol. 82, pp. 253-284, 1991.

[11] J. Westerhoff, "Defining 'Ontological Category'," Proceedings of the Aristotelian Society, vol. 102, pp. 337-343, 2002.

[12] H. A. Simon, "The Architecture of Complexity," Proceedings of the American Philosophical Society, vol. 106, pp. 467-482, 1962.

[13] J. Kohlas, "Uncertainity and Information," in An Introduction to Information Theory and Applications, F. C. Bavaud, J. C.; Kohlas, J., Ed., ed, 2005, pp. 9-80.

[14] G. Guizzardi, "Ontological Foundations for Conceptual Part-Whole Relations: The Case of Collectives and Their Parts," in Advanced Information Systems Engineering. vol. 6741, H. Mouratidis and C. Rolland, Eds., ed: Springer Berlin / Heidelberg, 2011, pp. 138-153.

[15] V. Ahl and T. F. H. Allen, Hierarchy theory, a vision, vocabulary and epistemology. New York: Columbia University Press, 1996.

[16] A. Tversky, "Features of Similarity," in Psychological Review, 1977, pp. 327-352.

[17] P. Simons, Parts: A Study in Ontology: Oxford University Press, USA, 1987.

[18] A. Varzi. (2011). Mereology. Available: http://plato.stanford.edu/archives/spr2011/entries/mereology/

[19] B. A. Davey and H. A. Priestley, Introduction to Lattices and Order. Cambridge, UK: Cambridge University Press, 1990.
[20] M. Winston, R. Chaffin, and D. Herrmann, "A Taxonomy of Part-Whole Relations," Cognitive Science, vol. 11, pp. 417-444, 1987.

[21] M. Bunge, Emergence and Convergence: Qualitative Novelty and the Unity of Knowledge: University of Toronto, 2004.

[22] K. Koffa, Principles of Gestalt Psychology. London: Routledge \& Kegan Paul Ltd, 1935.

[23] J. P. Crutchfield, "Is anything ever new?: considering emergence," in Complexity, ed: Perseus Books, 1999, pp. 515-537.

[24] T. O'Connor, "Emergent Properties," American Philosophy Quarterly, vol. 31, p. 14, 1994.

[25] A. J. Ryan, "Emergence is coupled to scope, not level: Research Articles," Complex., vol. 13, pp. 67-77, 2007.

[26] M. A. Bedau, "Weak Emergence," Philosophical Perspectives: Mind, Causation, and World, vol. 11, pp. 375-399, 1997.

[27] D. J. Chalmers, "Strong and weak emergence," in The Re-Emergence of Emergence, P. Davies and P. Clayton, Eds., ed: Oxford University Press, 2006.

[28] P. Laplante, Requirements Engineering for Software and Systems. Redmond, WA: CRC Press, 2009.

[29] E. Iee, "IEEE Recommended Practice for Software Requirements Specifications," IEEE Std 830-1998, 1998.

[30] D. Schon and G. Wiggins, "Kinds of seeing and their functions in designing," Design Studies, vol. 13, pp. 135-156, 1992.

[31] J. Gero and H. Jun, "Visual semantics emergence to support creative designing: a computational view," Department of Architectural and Design Science, The University of Sydney 1995.

[32] P. Gardenfors, Conceptual spaces: the geometry of thought. Cambridge, Massachusetts: The MIT Press, 2000.

[33] R. Jackendoff, Semantics and cognition. Cambridge, Massachusetts: The MIT Press, 1991

[34] J. A. Fodor, The language of thought. Cambridge, MA: Harvard University Press, 1975.

[35] S. E. Palmer, "Fundamental aspects of cognitive representation," in Cognition and Categorization, E. Rosch and B. Lloyd, Eds., ed: Lawrence Erlbaum, 1978, pp. 259-303.

[36] C. Larman, Applying UML and patterns. Upper Saddle River, NJ: Prentice Hall PTR, 2005.

[37] Erich Gamma, Richard Helm, Ralph Johnson, and J. Vlissides, Design Patterns: Elements of Reusable Object-Oriented Software. US: AddisonWesley, 1994.

[38] S. E. Sampson, "Customer-supplier duality and bidirectional supply chains in service organizations," International Journal of Service Industry Management, vol. 11, p. 16, 2000.

[39] R. F. Lusch, S. L. Vargo, and G. Wessels, "Toward a conceptual foundation for service science: contributions from service-dominant logic," IBM Syst. J., vol. 47, pp. 5-13, 2008.

[40] S. Alter, "Service system fundamentals: work system, value chain, and life cycle," IBM Syst. J., vol. 47, pp. 71-85, 2008. 